\title{
Locating Atoms in Small Crystals by Combining Convergent Beam Electron Diffraction and Electron Channeling
}

\author{
J. Tafto, S. Foss, A.Olsen and C. Simensen• \\ Department of Physics, University of Oslo, P. O. Box 1048 Blindern, 0316 Oslo, Norway \\ -SINTEF Materials Technology, P. O. Box 124 Blindern, 0324 Oslo, Norway
}

With the current focus on nanotechnology and nanomaterials, there is a growing interest for quantitative determination of composition and atomic arrangement within small volumes of materials. In chemical microanalysis the fast electrons within a subnanometer probe may excite inner shell electrons with accompanying element-characteristic energy losses and $\mathrm{x}$-ray emission that originate in the electron-illuminated volume of a thin specimen. The change, under channeling conditions, of the yield of element characteristic $\mathrm{x}$-ray emission was first observed by Duncumb [1], and the first observation in energy loss spectroscopy was done at Fritz-Haber-Institut [2]. These channeling effects, now often referred to as ALCHEMI, can be used to locate small concentrations of atoms within the unit cell of small crystals [3].

Convergent beam electron diffraction, CBED, is another powerful technique, although not necessarily quantitative for very small strained crystals of irregular shape for which the fine details associated with the dynamical interaction between incident beam and the microcrystal are lost. However, when the reflections with short g-vectors are far from the Bragg position, while several reflections well out in reciprocal space satisfy the Bragg condition, we come a long way with simple kinematical considerations. A CBED experiment that fulfills these constraints can be realized by tilting the specimen of the order one degree away from symmetrical incidence relative to a reciprocal lattice row [4].

The two techniques of ALCHEMI and CBED at large scattering angles are complementary. With ALCHEMI we are often able to determine the sites of the different elements within the crystal unit cell even when they are neighbors in the periodic table, but the accuracy with respect to coordinates for general positions may be limited to 0.05 $\mathrm{nm}$, while CBED with tilted crystal is capable of determining the position with an accuracy of $0.0005 \mathrm{~nm}$ [4].

As an example we have combined these two techniques in studying the quaternary intermetallic $\pi$-phase, a phase frequently encountered as thin platelets in aluminium alloys. Perlitz and Westgren [5], in their excellent x-ray diffraction work dating back to the forties, arrived at the composition $\mathrm{Al}_{8} \mathrm{Si}_{6} \mathrm{Mg}_{3} \mathrm{Fe}$, but they pointed out that the composition may deviate slightly from this value. The unit cell is hexagonal with $\mathrm{a}=0.664$ $\mathrm{nm}$ and $\mathrm{c}=0.7902 \mathrm{~nm}$. We restrict this presentation to the structural modulation along the c-axis where Perlitz and Westgren determined the sequence of atom planes shown in fig. 1. In fig. 1 is also included a calculated electron density modulation with the incident electron beam parallel to these atom planes, suggesting enhanced x-ray emission from iron, in agreement with our ALCHEMI experiments. The ALCHEMI results were in reasonable agreement with the structure reported in [5], but our experiments indicate some interchange of elements on the 6 inequivalent crystal sites, a possibility that was 
also was held open in ref. [5]. Fig. 2a shows a diffraction pattern with the wide convergent beam tilted away from normal to the $\mathrm{c}$ axis, and fig. 3 a scan across the reflections that are simultaneously at the Bragg position. Fig. 4 shows the calculated diffraction profiles. With the z-parameters of ref. [5] and the same Debye-Waller factor for all atoms, the agreement with observation is not good. However, by increasing the Debye-Waller factors for the atoms at general coordinates with $\mathrm{z}$ close to 0.25 , the $\mathrm{z}$ coordinates determined in ref. [5] agree well with our observations.

[1] P. Duncumb, Philos. Mag. 7 (1962) 2101

[2] J. Tafto and G. Lehmpfuhl, Ultramicroscopy 7 (1982) 287

[3] J. Tafto and J. C. H. Spence, Science 218 (1982) 49

[4] J. Tafto and T. H. Metzger, J. Appl. Cryst. 18 (1985)110

[5] H. Perlitz and A. Westgren, Arkiv Kemi, Mineralogi och Geologi 15 no 16 (1942) 1

[6] J. T. would express his gratefulness to G. Lehmpfuhl, E. Zeitler and the electron microscopy group at Fritz-Haber-Institut for great hospitality and inspiration while he stayed there. In part financed by the Norwegian Research Council under the FIN project .

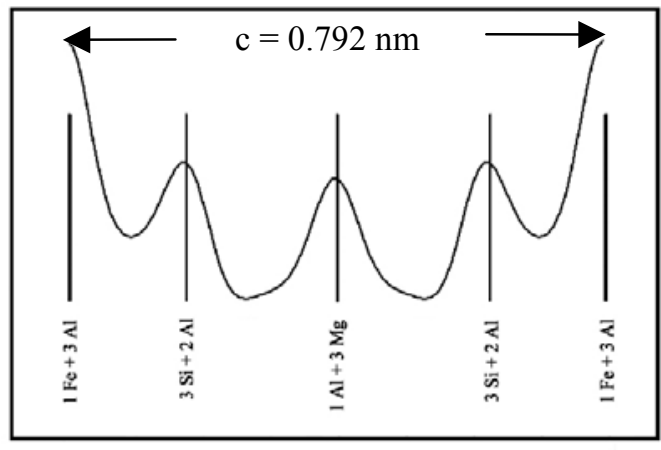

Fig. 1. The sequence of atom planes along the c-axis with calculated wave-field modulation.

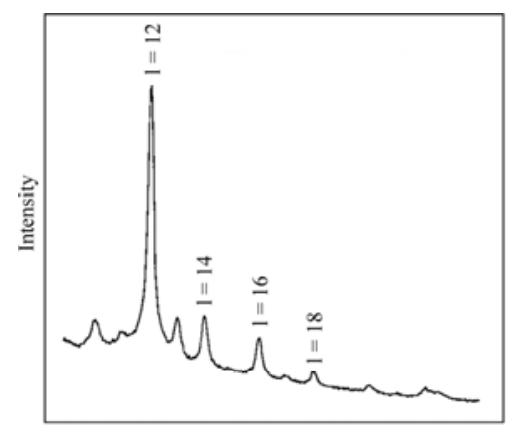

Fig. 3. Intensity profile across the reflections at Bragg position in fig. 2

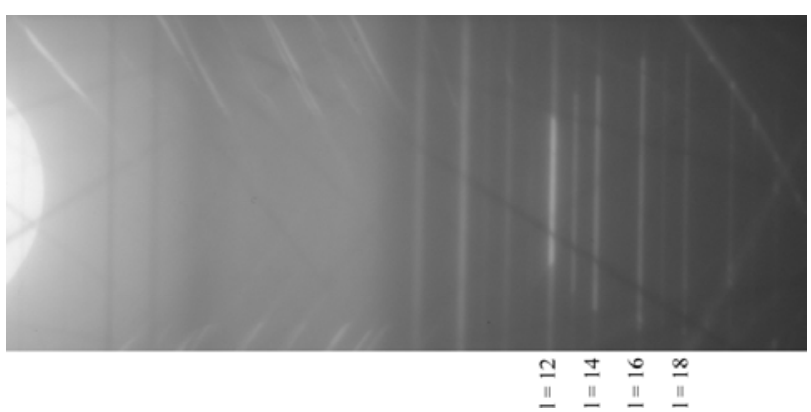

Fig. 2. CBED pattern with the reflections $0012-0021$ satisfying the Bragg condition.

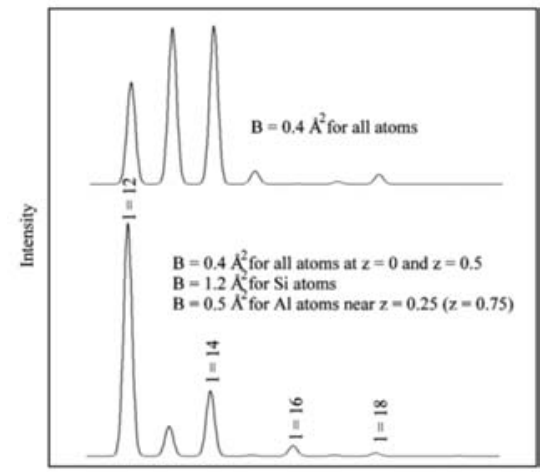

Fig. 4. Calculated intensity profiles with z- parameters from ref. [5] and two sets of Debye-Waller factors, B. 